\title{
ACTITUDES QUE DESARROLLAN LOS ALUMNOS DE SECUNDARIA AL CREAR COMPOSICIONES MUSICALES EN GRUPO $M^{\mathrm{a}}$ Ángeles Oriol López
}

\author{
Colegio Montpellier \\ mangelesorlo@yahoo.es
}

La música en educación secundaria puede ser un medio importante para desarrollar actitudes personales en los adolescentes. En el presente trabajo he tratado de comprender, a través de una investigación cualitativa, el proceso de aprendizaje y las actitudes que desarrolló un grupo de alumnos al crear composiciones musicales cooperativamente. La autoestima, la motivación y la socialización fueron los principales aspectos de la indagación, realizada mediante una estrategia de investigación-acción con la finalidad de mejorar mi propia práctica docente.

Music in secondary schools can be an important way to help adolescents develop personal attitudes. In this study I have tried to understand, through a qualitative investigation, the learning processes and the attitudes that a group of students developed when creating musical compositions collaboratively. Self-esteem, motivation and socialization were the main issues of the inquiry, which I carried out with an action-research strategy in order to my own teaching practice.

Las clases de música impartidas en los institutos de secundaria han estado centradas en la historia de la música y la teoría musical, siguiendo los libros elaborados por las editoriales, y utilizando una metodología fundamentalmente teórica en vez de emplear la práctica musical. Aún hoy es posible encontrar profesores de secundaria que siguen ese tipo de enseñanza. Para el alumnado actual esto suele ser motivo de actitudes negativas, derivadas del aburrimiento y la desmotivación. "Una práctica pedagógica como ha sido en general la del sistema, es decir, una enseñanza basada más en el conocimiento teórico de la música que en el desarrollo de capacidades perceptivas, expresivas y comunicativas o creativas, puede llevar al alumnado al aburrimiento y a la total falta de interés" (DÍAZ, 2005:69).

En este trabajo exploro una alternativa para mejorar el proceso de enseñanza-aprendizaje musical. Intento favorecer situaciones de aprendizaje práctico de la música que motiven y generen actitudes positivas en el alumnado. Al mismo tiempo, como profesora investigo mi propia práctica docente a través de una estrategia de investigación-acción. Los interrogantes de la investigación responden a unas inquietudes y preocupaciones. Por una parte, la situación tradicional de la música en educación secundaria. En segundo lugar, el convencimiento de que la música puede contribuir a la formación integral de la persona. Por ello los objetivos fueron observar hasta qué punto una metodología basada en la práctica musical en grupo, más que en la teoría, podía favorecer la motivación y el aprendizaje de cada alumno y, por otro lado, comprender qué actitudes podían desarrollar los estudiantes al tener que crear composiciones musicales en pequeños grupos. Las cuestiones que guiaron la indagación fueron:

- ¿En qué actitudes se ha expresado la autoestima del alumnado al desarrollar esta actividad?

- ¿QQué aspectos fueron motivadores y en qué actitudes se manifestaba dicha motivación?

- ¿Cómo ha contribuido la práctica musical en grupo a la socialización?

La finalidad de este trabajo ha sido, entonces, doble. Por un lado, contribuir al conocimiento pedagógico-musical. Por otro, mejorar mi propia práctica docente, mediante un trabajo de investigación-acción musical.

\section{FAVORECER UN APRENDIZAJE PROCEDIMENTAL Y COOPERATIVO}

Una didáctica centrada en la dimensión práctica de la música, en que el alumnado de secundaria componga, interprete y escuche música en lugar de recibir únicamente conceptos de musicología, puede ser más motivadora y por tanto, generar actitudes personales positivas que repercutan de forma adecuada en su autodesarrollo así como en el contexto general del aprendizaje. El profesor, como guía educativo, es el que debe potenciar el aprendizaje del alumno. Una alternativa metodoló- 
gica para ello sería favorecer aprendizajes más procedimentales que declarativos, más cooperativos que individuales en todos los niveles de la enseñanza. Del mismo modo que a caminar se aprende caminando o a hablar, hablando, a "musiquear" (ELLIOT, 1997) se aprende haciendo música. Esto plantea la necesidad de enseñanzas más prácticas que teóricas. O mejor, teorías que generen práctica musical y viceversa, práctica musical que genere teorías.

Para esta propuesta didáctica es útil tener en cuenta que las conductas de los alumnos, más que conductas son acciones significativas, entendiendo por éstas "la conducta física más las interpretaciones de significado del estudiante y de aquellos con quienes éste interactúa" (ERICKSON, 1989:214). Una forma de comprender esas acciones es potenciar el aprendizaje cooperativo en el aula de música. El aprendizaje cooperativo es muy importante en el aula a todos los niveles, pero especialmente con los adolescentes, que encuentran en el grupo un apoyo tanto académico como afectivo. Este aprendizaje fomenta fundamentalmente el diálogo y el enriquecimiento personal mediante aportar y recibir, como lo plantea BRUNER (1997:38) en su postulado interaccional, "los aprendices se ayudan a aprender unos a otros, cada cual de acuerdo con sus habilidades”. Además, puede ser una estrategia excelente para el desarrollo de actitudes como el respeto, la tolerancia y la cooperación.

Es importante no perder de vista un aspecto fundamental en la enseñanza de cualquier área curricular y, sobre todo, de la música, la motivación para aprender. Entiendo la motivación como el impulso que hace que pongamos todas nuestras energías para lograr algo. Y ese impulso, que implica esfuerzo y superación de dificultades, al mismo tiempo produce satisfacción cuando se obtienen resultados favorables. Una persona se esfuerza voluntariamente por algo, cuando para ella tiene sentido. En definitiva, cuando está motivada. Es necesario, por tanto, un planteamiento serio sobre lo que deberíamos modificar o incluir en la enseñanza, para neutralizar los riesgos del aburrimiento y de la pereza intelectual y emocional. El tema de las actitudes y de los valores no puede permanecer ajeno a ningún tipo de educación. Los valores nos educan, en el sentido de que tiran de nosotros y nos fuerzan a superarnos en una especie de flujo ascendente de auto-crecimiento, ELLIOTT (1997:24), innato a la persona, que debemos potenciar en vez de anular.

La investigación educativa-musical ha aumentado en las últimas décadas, como muestran las muchas recensiones realizadas (COLWELL, 1992; COLWELL y RICHARDSON, 2002; BRESLER, 2007) y la cantidad de revistas científicas dedicadas a ella. Sin embargo, el campo de las actitudes personales que desarrolla la música escolar no ha sido suficientemente investigado. En particular, este estudio se centra en las actitudes de los alumnos de secundaria cuando se emplea una metodología didáctica que pone el acento en la dimensión práctica de la música más que en la teoría.

\section{METODOLOGÍA Y DISEÑO DE LA INVESTIGACIÓN}

EISNER (1998) sostiene que hay que aprender a ver en profundidad y no sólo mirar la realidad educativa. Pero conocer más a fondo el mundo de las escuelas y de las aulas exige intentar comprender los procesos de aprendizaje que se dan en relación con cada contexto, ya que ningún aula es semejante a otra. El mundo de interrelaciones que se desarrolla entre las personas es tan complejo que su medición estadística aporta menos a la mejora educativa que la "indagación sistemática y autocrítica” propuesta por STENHOUSE (1987: 28) basada en la curiosidad y el deseo de comprender la realidad que tiene el profesorado. La investigación hecha por enseñantes requiere un trabajo detenido de observación y una recogida de datos sistematizada.A través de la auto-observación del trabajo de enseñanza-aprendizaje muchas veces se necesita modificar aquello que se considera poco eficaz para conseguir los fines propuestos. Por tanto, existiría una mejora de la práctica educativa que se va realizando "sobre la marcha". La expresión coloquial "sobre la marcha" supone un nivel de indeterminación en la enseñanza así como un planteamiento de las programaciones como algo abierto y en continuo cambio. La idea del profesor investigando la enseñanza exige de él la capacidad de ver la acción educativa como hipotética y experimental, nunca como una realidad cerrada e inamovible. En la estrategia de investigación-acción, la investigación orienta la acción, con- 
virtiéndose ésta en un modo sistemático de indagación y desarrollando una teoría que pueda ser comprobada por los profesores en las aulas (STENHOUSE, 1987).

El proyecto fue desarrollado con cuatro grupos de alumnos de $1^{\circ}$ de educación secundaria obligatoria (12-13 años de edad) en un centro concertado de Madrid. La estrategia didáctica consistió en la creación de composiciones musicales en grupos, que los propios alumnos establecieron, y su simbolización con partituras no convencionales. Para esta actividad se fueron estableciendo algunas pequeñas condiciones, como que su duración debía ser de al menos un minuto y que tenía que ser totalmente original, es decir, creada en su totalidad por los propios integrantes del grupo. La "acción” educativa fue llevada a cabo a lo largo de siete sesiones lectivas, y culminó con la interpretación de las composiciones en un concierto al que asistió el resto de los alumnos de $1^{\circ}$ de ESO y sus tutores.

Presenté la investigación a los alumnos como un reto, informándoles desde el principio de lo que se iba a realizar, y solicité el apoyo y la colaboración de sus tutores. Para tener una autorización formal, presenté un escrito a la dirección del centro y solicité un permiso al consejo escolar. La recogida de datos se hizo uso mediante diversos medios técnicos, desde la recogida de notas manuscrita hasta las grabaciones de audio, la filmación en vídeo o la fotografía. Las grabaciones de audio se emplearon en algunas entrevistas. Las tomas de vídeo y las fotos se realizaron durante el proceso de composición y en el concierto final. Las técnicas utilizadas fueron:

- Observación: de la presentación de la actividad de composición musical, de las sesiones de trabajo por grupos para la elaboración y el ensayo de la composición, de la presentación del proyecto inicial de cada grupo ante el resto de compañeros, del ensayo general en el aula en la quinta sesión de trabajo incluyendo una presentación y explicación de la partitura, y del concierto final en el salón de actos del centro.

- Entrevistas abiertas a alumnos individuales, entrevistas semiestructuradas en pequeños grupos, entrevistas estimuladas por un material gráfico (las partituras elaboradas), entrevistas estimuladas por vídeo (BURNARD, 2004) en gran grupo utilizando filmaciones de las distintas sesiones de trabajo y del concierto, entrevistas estimuladas por vídeo a los tutores, y entrevista abierta al director del centro.

- Notas de campo, registrando en un diario todo lo que iba ocurriendo en cada una de las sesiones de trabajo y en el resto de actividades.

- Cuestionarios de opinión pasados a los alumnos.

Para el análisis, en primer lugar se trascribieron los datos con un procesador de texto, y mediante comentarios al margen se fueron resaltando temas específicos. En un segundo momento, se trascribieron algunas expresiones a una hoja de cálculo, agrupándolas por categorías. Para garantizar la calidad del estudio se utilizó una triangulación de métodos y una triangulación de informantes.

\section{ANÁLISIS DE LOS DATOS}

\section{Actitudes relacionadas con la autoestima}

La autoestima, según ELLIOTT (1997:23), “se manifiesta como un sentimiento de que uno es exitoso, capaz o productivo”. En la investigación que presento se han analizado primero las actitudes relacionadas con la baja autoestima, y a continuación el modo en que se ha desarrollado positivamente la misma.

La falta de autoestima en los preadolescentes, está muy asociada a la inseguridad, como he podido observar en la investigación. Se ha manifestado en actitudes como necesidad de valoración continua, excesivo protagonismo manifestado en acciones para llamar la atención, preocupación por la calificación y dependencia de la misma para actuar de un modo u otro, timidez, miedo escénico, expresiones negativas (del tipo "no puedo", "no soy capaz”, "no me va a salir” o "yo no valgo") y necesidad de reconocimiento por parte de la familia. Esta actitud fue patente en un grupo de alumnos de rendimiento académico bajo, muy motivados con el trabajo de composición musical porque 
podían tocar y experimentar sonidos con diversos instrumentos. Pese a ser conscientes de que lo estaban haciendo bien, al terminar la cuarta sesión de ensayo se acercaron y me dijeron:

Ponnos una nota en la agenda para que sepan nuestros padres que lo estamos haciendo muy bien.

A la hora de opinar sobre las actuaciones de los otros grupos observé la baja valoración de los otros, resaltando siempre lo que hacían mal. Pocos resaltaban aspectos positivos, lo cual puede considerarse como expresión de baja autoestima en preadolescentes que se están desarrollando como personas y todavía no se aceptan a sí mismos.

El miedo escénico ante el concierto final, la vergüenza de actuar ante los compañeros e incluso ciertos prejuicios, resaltan en esta entrevista grupal:

-Cuando hagamos la actuación con todos [se refiere al concierto final] nos van a abuchear.

-Hagas lo que hagas te van a abuchear.

-No sabemos lo que han hecho otras clases... ¡Sorpresa!

-Cuando toco en público me entran nervios. Me tendré que controlar.

-Algunos me han dicho que parecíamos monos, por los bongos.

El miedo escénico les dificultó la interpretación a un grupo que había mostrado a lo largo de todas las sesiones mucha necesidad de llamar la atención, afectividad adolescente desbordante, variabilidad de estados anímicos y, en el trabajo, muchos cambios en la composición. Una de las integrantes se expresó del siguiente modo:

Pues que en el ensayo lo teníamos muy bien hecho y luego, al salir ahí, nos pusimos nerviosas y se nos olvidó todo.

Otra alumna, cuyo grupo compuso un rap, actuó en el concierto con la mano en la garganta. Su gesto fue considerado como una muestra de inseguridad y timidez. Durante una entrevista estimulada por el video del concierto sus compañeros le preguntaban:

- ¿Por qué te pusiste la mano en la garganta? [la alumna no contesta, se oye murmullo]

-Se ponía la mano en la garganta y quiero saber por qué y quiero saber por qué [se oye mucho jaleo y bromas]

Un aspecto que fortalece la autoestima es la satisfacción personal que produce el reconocer cualidades así como el gozo al obtener resultados positivos por el trabajo realizado. Un alumno dijo al finalizar la actividad:

Nunca olvidaré lo que he sido capaz de componer.

Los datos muestran relación entre autoestima y motivación: varios alumnos, al estar satisfechos con lo que hacían, se sentían más motivados intrínsecamente para continuar la actividad y, en la medida que iban obteniendo resultados positivos, se iban sintiendo más contentos.

Hay un aspecto que considero necesario destacar de la observación del alumnado; se trata de la relación entre satisfacción personal, que la entenderé como motivación intrínseca; los resultados académicos, como motivación extrínseca; y el nivel de exigencia personal o nivel de aspiración de las personas. Existe una relación directa: a más nivel de aspiración algunos alumnos necesitaban mejores resultados para sentirse satisfechos y viceversa, cuando el nivel de aspiraciones era más bajo, la satisfacción la obtenían con peores resultados. Por ejemplo, observé algunos alumnos que su satisfacción estaba relacionada con un esfuerzo grande de perfección en la composición que asociaron con un sobresaliente, sin embargo, otros, con un nivel de aspiraciones inferior, se conformaron con que la actividad estuviera medianamente aceptable y con poder aprobar. Y esto lo percibí fundamentalmente al realizar la autoevaluación; comenzaron primero por autocalificarse, ya que la pregunta que dirigí a los grupos fue: “¿Qué nota os pondríais y por qué?” Algunas de sus expresiones son:

-Yo me pondría un 7. No sé, porque no toco mucho, pero estoy satisfecho.

-Un 7, porque hemos cambiado mucho lo que íbamos a hacer.

-Un 8, porque he trabajado mucho y he hecho el final de la canción; el título de la canción se me ha ocurrido a mí. 
-Pues un 9 porque he estado trabajando siempre y he hecho todo.

-Un 10 porque la partitura la hemos tenido que completar y es lo que más difícil nos ha resultado. Creo que ha estado muy bien.

Todos los ejemplos expuestos cuantifican numéricamente la autovaloración del proceso de trabajo. Es evidente que no se refieren sólo al rendimiento final sino que tienen en cuenta su aportación al grupo así como otras actitudes relacionadas con el esfuerzo y la creatividad. Como ya indiqué anteriormente, es grande la influencia del sistema educativo que califica para evaluar y yo, integrada en el sistema, lo promuevo. Pero no cabe la menor duda de que están realizando un juicio valorativo autopersonal que refleja su autoimagen, al tiempo que repercute en su autoestima.

Hay un hecho concreto que se relaciona con la autoestima y es el modo de comportarse los alumnos que tienen estudios de música fuera de los escolares. En algunos casos han sido los líderes de su grupo. Optaron por tocar en el grupo en vez de componer solos. Observé que son estudiantes generalmente tímidos y que no sobresalen afectivamente aunque en sus calificaciones de las diversas áreas escolares sí suelen destacar. Puedo afirmar que la composición grupal fue una oportunidad de aportar cualidades personales.

En general, sus expresiones al ver la grabación final del concierto, expresan satisfacción por el logro conseguido. A algunos alumnos fue necesario animarles más que a otros y observé que los alumnos más independientes afectivamente y que obtienen buenos resultados, tienen un nivel de autoestima mejor y por consiguiente, su motivación intrínseca es mayor. Sin embargo, los alumnos más dependientes tienen la autoestima más baja y necesitan más atención y ánimo para salir adelante. Una alumna se expresa así al final:

Me ha gustado, aunque hemos estado haciendo un poco el vago, pero bueno..., pero al final hemos podido salir adelante porque tú nos decías que sacásemos algo porque siempre has estado ahí todo el rato diciéndonos que siguiésemos y que lo podíamos hacer aunque fuésemos un grupo numeroso y eso. Y que me ha gustado.

Otro reconoce que se lo ha pasado muy bien a pesar de los nervios del concierto. Considero que la actitud de saber disfrutar con una actividad como ésta, es una expresión de interés y motivación, que promueve la sana estima de uno mismo.

Pues que ha estado bien porque en los ensayos nos lo hemos estado pasando muy bien y luego, aunque nos hayamos puesto muy nerviosos en la actuación, también nos lo hemos pasado muy bien.

El siguiente alumno realiza una valoración positiva no sólo de su propio grupo sino de todos, lo que sería una expresión de su adecuada estima en este aspecto.

Que a mí me ha gustado mucho cómo nos ha quedado la actuación, pero que a todos los grupos les ha quedado muy bien.

Una alumna está muy satisfecha con su actuación y con el aprendizaje personal obtenido:

He visto que lo hemos hecho muy bien y que esto lo que nos ha enseñado ha sido a formar músicas por nosotros mismos, no hacer un baile con la música que conocemos, no, haciéndola por nosotros mismos.

\section{Actitudes relacionadas con la motivación}

El concepto de motivación empleado se refiere a los aspectos más afectivos. Motiva lo que produce satisfacción o resulta agradable. La motivación es intrínseca cuando el lograr resultados favorables se convierte en incentivo, o en reto, o cuando una persona se esfuerza por la superación personal (PINTRICH y SCHUnK, 2006). Asimismo se ha tenido en cuenta la necesidad de autorrealización que existe en cada persona, y que impulsa al logro de metas cada vez más altas. Desde este punto de vista un alumno estará motivado para aprender cuando disfrute con lo que está haciendo y además sienta que lo que hace es "efectivo" en el sentido de que da resultados positivos. Esa sensación de logro, identificable con el concepto de "dinamismo interior” del que habla ELLIOTT (1997), produce una enorme satisfacción personal convirtiéndose al mismo tiempo en impulso que le llevará a esforzarse por lograr sus objetivos. A continuación se presentan las principales actitudes aso- 
ciadas a sensaciones personales o estados de ánimo, observadas en el proceso de trabajo de los alumnos.

El interés general apareció relacionado con actitudes como: la prontitud y agilidad para ponerse a trabajar en la composición, la aportación de ideas, la expresión de sus rostros, la posición del cuerpo, etc. El entusiasmo se manifestó también en el deseo de que llegara la hora de la clase de música para trabajar en la composición que cada grupo iba elaborando. En general, la sensación de haberse divertido predominó hasta el final. A veces la sensación de diversión apareció asociada con un cierto estado de "relajación” producido por la actividad; entendiendo por "relajación”, un estado de distensión de las clases formales que se imparten en el aula.

-Sí, porque te relaja mucho y es entretenida.

-Colaboras y te lo pasas bien.

-Divertido porque lo hemos hecho todos juntos.

Uno de los tutores, en la entrevista estimulada por el video de los ensayos de los alumnos, se fijó en un grupo de alumnos bastante complicados y me dijo:

Es importante que no estén descontrolados, haciendo el vándalo, tonterías, dando golpes, sino que están allí experimentando, a lo suyo; han tenido como que pasar la racha anterior, de más movimiento, pero ahora están bastante bien. Significa que lo están disfrutando un poco.

Varios alumnos expresaron de diversos modos que el hecho de haber compuesto juntos, fue un aliciente para seguir adelante. Es una muestra de la importancia que tiene en la motivación el aspecto de la socialización.

-Cuando algo no le salía a alguno le hemos ayudado y lo hemos logrado.

-He aprendido que la música en grupo es más divertida que si la toca uno solo.

No obstante, algunos grupos, encontraron más dificultades en esta actividad y, en consecuencia, se sintieron menos satisfechos y entusiasmados. Incluso para alguna alumna, en la realización de esta actividad se mezcló el agobio con la diversión.

Ha sido la primera vez que nos hemos puesto a sacar notas de una canción y me ha resultado, a ratos, agobiado y a ratos divertido.

Al expresar su motivación por esta actividad, es interesante destacar la crítica que realizaron, sin apenas ser conscientes de ello, al sistema educativo y en concreto, a un tipo de metodología poco activa en las clases convencionales. Además sacaron en conclusión que "la música es divertida" porque se da de otro modo. Algunas de sus expresiones fueron:

-Es menos aburrido que las clases de siempre.

-Sí, lo hemos pasado bien. Nos hemos divertido. Estamos acostumbrados a dar clase sentados y eso es aburrido; y con esto hemos visto que la música es divertida.

La sensación de integridad, de satisfacción ante la obra bien terminada apareció en algunas composiciones de alumnos. Considero interesante aportar lo que una tutora comentó en la entrevista apoyada en el video que se le realizó, tras ver la actuación del primer grupo de su clase.

Me parece, primero, que los alumnos están en una actitud cómoda porque los veo sonrientes, lo que quiere decir que eso les ha satisfecho. La forma en la que han organizado la música es como una música primitiva que es el origen de todo, de manera que ellos se han basado en la percusión de alguna forma ¿no? Y han seguido un orden, una presentación, a través de esas maneras y luego han hecho una culminación y un final, me gusta eso, que tenga una estructura. En ese sentido, pues está bien. Me ha gustado.

La mayoría de los grupos, trabajaron con libertad porque podían hacer lo que querían. Eso les motivó y generó actitudes de autonomía y autorregulación, que se reflejaron en el hecho de organizarse con rapidez y trabajar aprovechando el tiempo, con satisfacción e interés, así como el que no fuera necesario estar diciéndoles en cada momento lo que tenían que hacer. Esto fue también motivo de gozo y satisfacción.

-Haces una canción que es a tu gusto.

-Me ha gustado porque nos dejabas "por libre" 


\section{Y otro alumno comenta:}

-He aprendido a tocar los instrumentos y tocar el xilófono, que me encanta.

$-\dot{i}$ Antes te gustaba? -indago

-Antes no me dejaban.

-¿Nunca te dejábamos?

- No.

Y relacionaron claramente esa sensación de libertad con un tiempo de trabajo ameno y entretenido:

Ha sido más ameno porque hemos podido hacer lo que queríamos.

Es curioso cómo los alumnos reconocieron y valoraron la necesidad del esfuerzo al final, después de ver el resultado de su actuación en el video. De hecho, al principio, en los primeros ensayos, algunos reconocieron, que les faltó esfuerzo y seriedad. Incluso hay que destacar la dimensión solidaria del mismo como muy positiva.

-Nos ha costado mucho. Pero nos hemos esforzado y nos ha quedado bien.

-Había cosas difíciles que, ensayándolas, hemos conseguido saber hacerlas.

-Cuando algo no le salía a alguna, la hemos ayudado, y lo hemos logrado.

Incluso lo relacionan con la diversión y el aprendizaje en el sentido de que les mereció la pena esforzarse por lo bien que lo pasaron.

Ha merecido la pena el esfuerzo porque lo hemos pasado muy bien, imaginando canciones, notas, aprendiendo dónde poner las notas.

También la premura del tiempo es la que les ayudó a esforzarse para tener la composición terminada en la fecha indicada. Creo que es importante que los alumnos sepan que existen unos límites expresados a través de unas condiciones, a los que han de sujetarse.

Al principio vagueando y de juerga, pero luego, como había poquito tiempo, nos hemos esforzado y lo hemos hecho bien.

Y unos alumnos al ver la grabación del concierto y de los ensayos comentaron:

-Pues que todas estas grabaciones no las hemos hecho en un día sino que hemos tenido tiempo y nos hemos esforzado para poder hacerlas bien.

-Pues que yo creo que al principio empezábamos como así, de guasa, pero luego, empezamos ya a ponernos en serio y al final hemos conseguido hacer una canción, así que las cosas hay que hacerlas con esfuerzo porque si no, no salen.

-Pues yo creo que todas las cosas tienen su mérito porque por ejemplo, la nuestra aunque dura poco está currada.

-Nos hemos esforzado mucho y hemos hecho un buen trabajo, pero también ha habido momentos de guasa y nos ha gustado mucho porque hemos aprendido también música.

\section{Actitudes relacionadas con la socialización}

En el aspecto de la socialización he observado cómo a través de la música grupal, se desarrollan actitudes que favorecen la convivencia positiva y el compañerismo. Por "convivencia" entiendo la capacidad de relacionarse positivamente con los demás y con el entorno. Utilizaré el término como un valor que se manifiesta en diversas actitudes observadas en el proceso de trabajo del alumnado. Las principales actitudes han sido el correcto comportamiento durante el trabajo de composición relacionado con el respeto y la escucha así como el cuidar y compartir instrumentos.

Algunos alumnos expresaron en distintos momentos, que se portaban bien porque no estaban en clase aburridos, es decir, la positiva convivencia se encuentra muy relacionada con una didáctica “amena” y motivadora.

A mí me ha gustao mucho esta experiencia y quiero decir que este trimestre, nos hemos portado muy bien porque como hemos estado tocando los instrumentos y todo eso... pues no hemos estado en clase y no nos hemos aburrido; por eso nos hemos portado tan bien. 
En algunas ocasiones se produjeron discusiones con respecto a la distribución de los instrumentos, sobre todo al principio. En el siguiente texto de mis anotaciones se recoge un ejemplo.

Un grupo de niñas, con C. y M. al frente, como líderes positivos, trabajan en inventar melodías nuevas con la flauta y el carillón. Mientras, otras compañeras del mismo grupo protestan porque no tienen los instrumentos que están utilizando un grupo de chicos. Incluso "aporrean” en algún momento el pandero para hacerse notar y molestarles.

Me parece importante hacer una reflexión sobre la convivencia y los líderes. A veces el problema de convivencia no guarda relación directa de causa-efecto con la actividad musical realizada, sino que está influido por otros problemas más generales, como la presencia de un líder negativo. Tal es el caso de un único grupo que no actuó en el concierto final. Estos alumnos necesitan una atención más personalizada, que no siempre el profesor puede prestarle.

Por el contrario, puedo afirmar, que la presencia de líderes positivos en los grupos, favorece la convivencia y ayuda para que algunos alumnos rindan mejor. Los tutores hicieron referencia a este tema del liderazgo como un factor condicionante. Uno comentó:

Gracias al líder que hay en cada grupo, el grupo se puede formar, aunque hay grupos que se sienten "peorcillo", en la relación, otros mejor, pero es muy importante mirar dónde están los líderes. Parece que se unen entre ellos y los demás se quedan relegados ahí y no saben nada. Sin embargo aquí se han repartido bastante bien en cada grupo...

Y continuó señalándome los que consideraba líderes positivos de los grupos de su clase. Es una triangulación importante de mi observación porque coincide plenamente con los que yo había detectado.

Sin embargo, el tutor de otra clase se quejó de la falta de líderes definidos en su aula, ya que el presente curso fueron mezclados todos los alumnos procedentes de $6^{\circ}$ de primaria.

Yo precisamente creo que el gran problema que ha habido al mezclar los grupos es que han desaparecido los liderazgos. Entonces, son liderazgos más bien provisionales. Toma uno la iniciativa, pero no hay detrás un apoyo del líder fuerte.

Las relaciones de compañerismo se han expresado en el modo de agruparse, en la integración y relación entre los compañeros, y en el trabajo cooperativo que han realizado aceptando que unos compañeros trabajen menos que otros. Por "compañerismo" entiendo todas las relaciones positivas que se establecen en un aula entre los alumnos. Al dejarles que se organizaran libremente, la actividad resultó una especie de sociograma de los alumnos de cada aula. Generalmente, los chicos con más dificultades de convivencia se agruparon; los más estudiosos también. Otro dato es que, salvo algún grupo mixto, la mayoría del alumnado se agrupó por sexo y amistad; es una actitud bastante frecuente en esta edad preadolescente. Asimismo la integración de compañeros que no pertenecían al grupo de amigos, supuso dificultad en algún momento, pero al final fue positiva en la mayoría de los casos. Tengo que reconocer que en ocasiones, tuve que actuar de profesora-mediadora en pequeños conflictos que dificultaban el seguir adelante con el trabajo.

Bastantes alumnos expresaron en las entrevistas la ayuda mutua que existió para superar dificultades y lograr el trabajo final. Y he observado lo importante que es el que cada alumno haya aportado sus cualidades realizando aquello que sabe hacer mejor. Al final eso les produjo bastante satisfacción.

-Al principio estábamos mal organizados, no nos llevábamos bien y hemos conseguido organizarnos y nos ha salido bien.

-Al principio discutíamos pero nos hemos conseguido sincronizar.

-Al principio nos peleamos porque no salía nada, pero luego salió. Fue difícil. 


\section{CONCLUSIONES Y PROPUESTAS}

Para los estudiantes de secundaria, el aprender "haciendo música” en grupo favoreció actitudes que posiblemente no se habrían desarrollado del mismo modo si la asignatura se hubiera impartido teóricamente. El reconocer cualidades que no sabían que tenían y la valoración positiva no sólo de sí mismos sino de los demás, potenció el autoconcepto personal así como la obtención de resultados positivos por el trabajo realizado. La satisfacción producida por la obra bien hecha les sirvió como refuerzo de la propia seguridad y, en consecuencia, de la autoestima. Cuando algo les supone esfuerzo, lograrlo se convierte en un importante factor de satisfacción; por ello la elaboración de la partitura totalmente original y la actuación en el concierto final pueden considerarse medios para el desarrollo de la autoestima.

Por otra parte cabe decir que se detectaron actitudes de baja autoestima en algunos alumnos, como prejuicios afectivos infundados ante la actuación en público, y el miedo escénico, que produce vergüenza, nervios y bloqueo en la actuación pública. También demanda continua de atención y reconocimiento, excesivo protagonismo, problemas de disciplina, modales negativos y poca valoración del trabajo de los otros.

El interés, entusiasmo, satisfacción y disfrute con lo que iban realizando son una clara manifestación de la motivación. El trabajo en grupo fue un importante factor de motivación porque les generó actitudes como el apoyo y ánimo mutuo. Y el hecho de trabajar en un ambiente de cierta libertad, les motivó y generó actitudes de autonomía y autorregulación reflejadas en la actitud de organizarse con rapidez y trabajar aprovechando el tiempo, con satisfacción e interés, así como el que no fuera necesario estar diciéndoles en cada momento lo que tenían que hacer. Relacionaron claramente esa sensación de libertad con un tiempo de trabajo ameno y entretenido.

Las actitudes más relacionadas con la socialización, se pueden vincular a dos aspectos: la convivencia positiva y el compañerismo. El respeto y la escucha, así como el cuidar y compartir los instrumentos disponibles se puede considerar una manifestación de adecuada convivencia. Por otra parte, el trabajo cooperativo realizado facilitó muchas de las actitudes de compañerismo observadas. La principal, la ayuda mutua que existió y reconocieron para superar dificultades y lograr el trabajo final. Pero también se detectaron algunos problemas de relación como integrar y acoger a compañeros en el grupo que no formaban parte de su círculo de amistad. También tuvieron que aceptar con cierta dificultad, que dentro del grupo unos compañeros trabajasen menos que otros.

En definitiva, es de gran importancia que hayan caído en la cuenta de que la música es divertida y pueden inventar su propia música, aunque no sea tan buena. Si han disfrutado con esta actividad puede considerarse una experiencia significativa para ellos.

Para mejorar la práctica docente sería muy conveniente establecer en el aula actividades que favorezcan las relaciones humanas cordiales basadas en la valoración positiva y el respeto. Asimismo es necesario potenciar actividades en las que los alumnos se expresen públicamente ante los demás, para que fortalezcan su seguridad personal, y fomentar la mejora del proceso de aprendizaje del alumno, desde su propia autorreflexión.

Teniendo en cuenta los aspectos anteriores, propongo como temas de investigación que pueden enriquecer el conocimiento en general y específicamente, la educación musical, investigar el rol del profesor de música, observando fundamentalmente el tipo de relaciones que fomenta en su aula, y las actitudes que se generan en los alumnos. En segundo lugar, analizar qué tipo de actividades musicales, con expresión pública, suelen realizar los profesores de música en su actividad docente, y qué influencias tienen en los alumnos. Y por último estudiar el sistema de evaluación que emplean los profesores de música en sus aulas.

Estoy convencida de que el trabajo del profesorado investigando su propia práctica docente constituye impulso para la verdadera calidad educativa de los centros, además de un medio de enriquecimiento y desarrollo personal. Si esto se promoviera e institucionalizara, seguramente la educación sufriría una gran revolución a través de las aportaciones de los profesionales de la enseñanza. 


\section{Referencias}

BRESLER, L. [ed.] (2007): International handbook of research in arts education. New York, Springer.

BRUNER, J. (1997): La educación, puerta de la cultura. Madrid, Visor.

Burnard, P. (2004): "Making musical learning visible: Using video stimulated reflection to examine the intersecting sound worlds of teacher-pupil thinking". En A. GIRÁLDEZ; M. J. Aramberri; F. BAutista; M. Díaz; L. Hentschke y M. Hookey (eds.): Proceedings from the 26th World Conference of the International Society for Music Education [cd-rom]. Madrid, Enclave Creativa.

Colwell, R. [ed.] (1992): Handbook of research on music teaching and learning. New York, Schirmer.

Colwell, R.; Richardson, C. P. [eds.] (2002): The new handbook of research on music teaching and learning. New York, Oxford University Press.

DíAZ, M. (2005): “Las improvisaciones infantiles como estrategia didáctica”. Eufonía. Didáctica de la Música, 35, 68-80.

DowLING, W. J. (1998): “Conocimiento procedimental y conocimiento declarativo en educación y cognición musical”. Orpheotron, 4, 23-40.

EISNER, E.W. (1998): El ojo ilustrado: Indagación cualitativa y mejora de la práctica educativa. Barcelona, Paidós

ElliotT, D. (1997): "Música, educación, y valores musicales". En V. Hemsy DE GainZA (ed.): La transformación de la educación musical a las puertas del siglo XXI. Buenos Aires, Guadalupe.

ERICKSON, F. (1989): "Métodos cualitativos de investigación sobre la enseñanza". En M. C. WiTTROCK (ed.): La investigación de la enseñanza, II. Métodos cualitativos y de observación (págs. 195-301). Barcelona, Paidós..

Hargreaves, D. J. (1998): Música y desarrollo psicológico. Barcelona, Graó.

PinTRICH, P. R.; SCHUNK D. H. (2006): Motivación en contextos escolares. Teoría, investigación y aplicaciones. Madrid, Pearson Educación.

STENHOUSE, L. (1987): La investigación como base de la enseñanza. Madrid, Morata.

Manuscrito recibido el 30/5/2007 y evaluado anónimamente. Aceptado para su publicación el 30/12/2009 